\title{
Guerre sainte dans le Cône sud latino-américain : pentecôtistes versus umbandistes
}

\section{Alejandro Frigerio et Ari Pedro Oro}

\section{(2) OpenEdition \\ Journals}

Édition électronique

URL : https://journals.openedition.org/jsa/3027

DOI : 10.4000/jsa.3027

ISSN : $1957-7842$

Éditeur

Société des américanistes

Édition imprimée

Date de publication : 5 décembre 2005

Pagination : 185-218

ISSN : 0037-9174

\section{Référence électronique}

Alejandro Frigerio et Ari Pedro Oro, «Guerre sainte dans le Cône sud latino-américain : pentecôtistes versus umbandistes », Journal de la Société des américanistes [En ligne], 91-2 | 2005, mis en ligne le 10 juin 2010, consulté le 02 septembre 2022. URL : http://journals.openedition.org/jsa/3027 ; DOI :

https://doi.org/10.4000/jsa.3027 


\title{
GUERRE SAINTE DANS LE CÔNE SUD LATINO-AMÉRICAIN : PENTECÔTISTES VERSUS UMBANDISTES
}

\author{
Alejandro FRIGERIO * et Ari Pedro ORO **
}

Dans ce travail, nous analysons la forme prise par le conflit existant entre l'Église universelle du royaume de Dieu et les religions d'origine afro-brésilienne au Brésil, en Uruguay et en Argentine. On soutient l'hypothèse que l'intensité de ce conflit est à mettre en relation avec les niveaux de légitimité et de visibilité sociale acquis par ces deux religions dans chaque société, ainsi qu'avec le degré d'organisation locale des afro-umbandistes, surtout avec l'existence, ou non, d'un cadre interprétatif d'action collective qui met en relief les droits civils. En outre, on s'interroge sur l'importance des variables attachées aux contextes qui transcendent les deux groupes religieux. Au niveau national, il apparaît que les discours dominants de la nation, ou de la région pour le Brésil, sont très importants dans chaque pays. Ces récits assurent en effet une place aux minorités religieuses à l'intérieur de la nation et constituent des ressources culturelles qui peuvent être mobilisées pour revendiquer les droits propres à chaque groupe ou pour attaquer leurs opposants. [Mots clés : guerre sainte, Amérique latine, pentecôtisme, umbanda.]

Holy war in the Latinamerican Southern Cone : pentecostalism versus umbanda devotees. The article examines the conflicts that have developed between the Igreja Universal do Reino de Deus and practitioners of Afro-Brazilian religions in Brazil, Uruguay and Argentina. It is argued that the intensity of their struggle is related to the degrees of social visibility and legitimation that these religions enjoy in each society as well as to the extent of organization that Umbanda devotees have attained - the existence of a collective action frame emphasizing their civil rights being particularly important in this regard. The paper also takes into account the relevance of contextual variables that trascend both religious groups, highlighting the role of the dominant national narrative that prevails in each country. Since this historical narrative assigns a specific place to the different ethnic and religious minorities within the nation, it constitutes a cultural resource that may be mobilized to vindicate a group's rights or to deny them to its opponents. [Key words : Holy war, Latin America, pentecostalism, umbanda.]

*Université catholique argentine/CONICET [Alejandro_Frigerio@il.com.ar].

** Université fédérale du Rio Grande do Sul, Av. Benito Gonçalves 9500, Bairro Agronomia 95.000.030, Porto Alegre, Brésil [arioro@uol.com.br].

Journal de la Société des Américanistes, 2005, 91-2, pp. 185-218. O Société des Américanistes. 
Guerra santa en el Cono Sur latinoamericano : pentecostales versus umbandistas. El trabajo analiza la forma que toma el conflicto entre la Iglesia Universal del Reino de Dios y las religiones de origen afrobrasilero en Brasil, Uruguay y Argentina. Se argumenta que la intensidad del mismo se relaciona con los niveles de legitimidad y de visibilidad social que ambas religiones alcanzan en cada sociedad y con el grado de organización local de los afro-umbandistas - particularmente, con la existencia o no de un marco interpretativo de acción colectiva que enfatice sus derechos civiles. Asimismo, se indaga sobre la importancia de factores contextuales que trascienden a ambos grupos religiosos. A nivel nacional, son particularmente importantes las narrativas dominantes de la nación - o de la región, en el caso brasilero - prevalentes en cada país. Estas narrativas asignan un lugar a las distintas etnias y minorias religiosas dentro de la misma y constituyen recursos culturales que pueden ser movilizados para reivindicar derechos propios o para negárselos al adversario. [Palabras claves : guerra santa, América latina, pentecostalismo, umbanda.]

Salvador de Bahia, Brésil, février 2004. Émission télévisée « Point de Lumière » (Ponto de Luz), retransmise par la Rede Record, sur tout le territoire national. Un téléspectateur appelle et raconte qu'il a trouvé sur le seuil de sa maison un petit sac contenant de la terre et une patte de poulet. Préoccupé par cette trouvaille, il demande ce que cela peut vouloir dire. Le pasteur qui présente l'émission parle à une personne installée derrière un paravent. Une lumière permet de voir une partie de sa silhouette : son torse et le profil de son visage. Les traits que l'on peut deviner ainsi que sa voix font penser qu'il s'agit d'une femme noire. Il demande à cette " $e x$-mãe de encosto $\|^{1}$ si elle peut expliquer à quoi sert ce travail spirituel. Elle répond que c'est un travail très dangereux, fait avec de la terre de cimetière, afin de provoquer la mort de celui qui le reçoit. À la question du pasteur-présentateur lui demandant si elle réalisait elle-même ce genre de travaux, elle répond qu'elle en faisait habituellement et qu'ils étaient très efficaces. Le pasteur invite alors celui qui a appelé, ainsi que d'autres téléspectateurs ayant des problèmes similaires, à se rendre au temple le lendemain, où 20 " ex-mães de encosto » recevront ceux qu'elles pensent pouvoir être victimes de sorcellerie et leur donneront des conseils sur la marche à suivre pour la combattre. Au Brésil, pratiquement n'importe quel individu est en mesure de comprendre que l'expression " ex-mãe de encosto » fait référence à une " ex-mãe de santo " (" mère-de-saint ») qui, désormais convertie à l'Église universelle du royaume de Dieu, pourra aider à défaire le mal qu'elle (la mère-de-saint) faisait auparavant.

\section{Buenos Aires, Argentine, mai 2004. Émission télévisée « Cessez de souffrir » (Pare de Sufrir).}

Un reportage résume les «sept façons pour un esprit malin de posséder une personne ». Selon le narrateur, « cela se produirait surtout par le contact avec des personnes qui vénèrent ces esprits ou avec des objets qui ont été offerts à leurs victimes. On peut notamment être possédé : par héritage familial ; par participation directe ou indirecte à des travaux de sorcellerie ; pour avoir été victime de 
travaux de sorcellerie ; à travers des nourritures sacrifiées aux idoles et par rejet du Christ ». Les références aux « esprits » ou aux « démons » sont en majorité illustrées par des films qui montrent des statues de plâtre d'Exu ou par des médiums d'umbanda en transe ; " les sorcelleries » le sont par des bougies que l'on allume devant des statuettes d'umbanda, par des mains tapant sur des tambours ou des images d'offrandes de nourritures, caractéristiques de cette religion. Les "personnes qui pratiquent la sorcellerie » - la façon la "plus habituelle » d'entrer en contact avec des esprits malins - sont représentées par des images de femmes portant des habits religieux blancs, typiques de l'umbanda, devant un autel de cette religion.

Montevideo, Uruguay, mai 2004. Émission télévisée « Cessez de souffrir ».

« Dans la macumba, ils font de la sorcellerie, dans la maison des encostos [esprits néfastes]. S'ils font des travaux avec les encostos pour détruire, nous, nous allons travailler encore plus fort ", dit le pasteur Santos avant d'inviter un fidèle, qui avait raconté sa terrible histoire par téléphone, à se rendre à l'église avec un bout de vêtement quelconque appartenant au membre de sa famille ensorcelé, afin de s'engager sur le chemin du bonheur. « Le sort sera défait ", promet le pasteur. Il a ensuite présenté de façon didactique « une fiction fondée sur un fait réel » pour que les infidèles voient comment agissent les " encostos ». Les écrans montrent alors un couple heureux, amoureux, ayant un travail et une bonne situation, qui trouve un jour sur le pas de sa porte des bougies allumées et des rubans rouges. On entend des tambours en musique de fond. La maîtresse de maison piétine le tout, visiblement effrayée, et au bout de quelques jours, les disputes font leur apparition au sein du couple, pour des broutilles. "Je n'ai pas d'argent, nous avons commencé à nous disputer, mon mari est malade, j'ai perdu mon travail. Que peut-il bien être arrivé ?», se demande la femme affligée, alors que l'on entend à nouveau le son des tambours, et que l'image des bougies et des rubans nous renvoie au passé. (Hebdomadaire Brecha, du 28 mai 2004, "El embate neopentecostal : con jabón en mano, y al umbandismo condenando » ${ }^{2}$ )

Au cours des dernières décennies, les religions afro-brésiliennes se sont développées aux niveaux social et spatial, en franchissant d'anciennes barrières de classe et de race, puisqu'elles ont réussi à se faire accepter par les couches moyennes et les différentes ethnies de la société brésilienne, ainsi que des barrières régionales et, plus récemment, des frontières nationales, puisqu'elles ont conquis un nombre significatif d'adeptes en Argentine et en Uruguay. Dans leur mouvement d'expansion, elles sont parvenues à des degrés divers de légitimité sociale, suscitant des alliances et des conflits avec différents acteurs sociaux. Seule la dynamique et puissante organisation néo-pentecôtiste brésilienne, l'Église universelle du royaume de Dieu (EURD), a atteint un développement supérieur puisqu'elle s'étend non seulement dans l'ensemble du Brésil et des pays limitrophes, mais a aussi acquis, dernièrement, une portée globale (Corten, Dozon et Oro, éds 2003). En se déployant dans le Cône sud latino-américain et en étant ainsi confrontée aux religions afro-brésiliennes, l’Église universelle a exporté la 
« guerre sainte » (Oro 1997) engagée sans pitié contre ces dernières dans leur pays d'origine.

Ce travail prétend analyser le conflit entre ces deux groupes religieux tel qu'il se déroule au Brésil, en Argentine et en Uruguay. Notre hypothèse est que l'intensité de ce conflit est liée aux niveaux de légitimité et de visibilité sociale atteints par ces deux religions au sein de chacune des sociétés considérées, ainsi qu'au degré d'organisation locale auquel sont parvenus les umbandistas ${ }^{3}$. Sur ce dernier point, l'existence ou non d'un " cadre interprétatif d'action collective » qui mette en avant leurs droits civils et la possibilité de mobiliser en leur faveur des " ressources culturelles » déterminées s'avèrent déterminantes ${ }^{4}$. L'une des ressources culturelles particulièrement importante serait les « constructions narratives nationales dominantes » prévalant dans chacun des pays - ou régions, dans le cas du Brésil - dans la mesure où, en attribuant une place et une valeur particulières aux différentes ethnies et minorités religieuses qui composent la nation ou la région, elles leur permettent de revendiquer leurs droits ou d'attaquer leurs adversaires (Frigerio 2002a) ${ }^{5}$. L'analyse des données à notre disposition montre que le conflit entre l'EURD et l'umbanda renforce le développement d'un champ social transnational, au sein duquel les umbandistas de ces trois pays se tiennent au courant, principalement via internet, des conflits qui se produisent avec l'EURD dans chacun de leurs pays. Les événements brésiliens, pays où la dispute a commencé et où elle s'est développée de la manière la plus virulente, ont eu une incidence particulière. Les querelles entre l'umbanda et l'EURD à Porto Alegre, dans l'extrême sud du Brésil, sont considérées - étant donné les relations étroites que les umbandistas du Rio de la Plata entretiennent avec les leaders religieux de cet État - comme de dangereux précédents. L'action menée par les umbandistas de la région du Plata, ces dernières années, vise en partie à éviter que les mêmes problèmes ne se reproduisent dans leurs pays.

Si la légitimité sociale acquise par les religions afro-brésiliennes tient dans une large mesure à leur degré d'insertion - en raison de leurs origines noires - dans les constructions narratives historiques dominantes de chaque nation ou région, pour l'EURD, en revanche, elle découle principalement de son récent succès dans l'arène politique. La quantité croissante de députés qu'elle a réussi à faire élire au congrès brésilien - seize députés et un sénateur en 2002 - lui a apparemment permis de faire oublier les dures controverses qu'elle avait suscitées dans les années 1990 (Giumbelli 2002). Au-delà de la préoccupation concernant les attaques de l'EURD dans ses émissions télévisées, ce pouvoir politique qu'elle a acquis inquiète les umbandistas des trois pays car il se pourrait bien que, sous l'influence des législateurs évangélistes, des lois viennent compromettre le libre exercice de leur religion.

Dans certaines régions du Brésil, où les religions afro-brésiliennes jouissent d'une forte légitimité de par leurs origines ethniques, l'inquiétude face au pouvoir politique et médiatique croissant de l'Église universelle a donné lieu à un mouve- 
ment de réaction contre ses attaques, mobilisant en leur faveur un large spectre d'acteurs sociaux dont des activistes et législateurs noirs. Un mouvement similaire a commencé à apparaître en Uruguay suite à une légitimation accrue de l'umbanda et à la reconnaissance tardive de l'existence d'une population afrouruguayenne. En Argentine, au contraire, où l'EURD est autant stigmatisée que les religions d'origine afro-brésilienne, bien qu'elle soit moins visible sur la scène sociale, le conflit a été de faible intensité. En s'appuyant sur ces prémices, nous tenterons de mettre en évidence les entrecroisements des dimensions raciales et politiques dans l'étude de la dynamique des mouvements religieux.

\section{Transnationalisation des religions brésiliennes. I. Le Cas de L'Église universelle du Royaume de Dieu}

Historiquement le Brésil a toujours été un pays importateur de religions. Au cours de ces trente dernières années, cependant, on constate également une autre tendance : celle de religions considérées comme « brésiliennes », pour être nées au Brésil, qui traversent les frontières nationales pour s'installer dans d'autres pays et continents ${ }^{6}$. Il s'agit surtout des Églises évangélistes néo-pentecôtistes et, en particulier, de l'Église universelle du royaume de Dieu ainsi que des religions afro-brésiliennes.

\section{La transnationalisation de l'Église universelle du royaume de Dieu}

Fondée en 1977 à Rio de Janeiro par Edir Macedo - un ancien employé de la fonction publique né en 1945 qui, dans son parcours religieux, était passé du catholicisme à l'umbanda avant de se convertir au pentecôtisme à l'âge de 18 ans - l'Église universelle est devenue le phénomène religieux le plus surprenant et le plus important de ces deux dernières décennies (Mariano 2003). Impressionnante en nombre ${ }^{7}$, cette Église constitue de surcroît, selon Harvey Cox (2003, p. 5), « le mouvement religieux le plus transnational du monde aujourd'hui... ».

En effet, le succès de cette Église «n'est pas celui d'une secte fermée sur elle-même, mais de conquérants de la foi d'un nouveau style qui n'ont pas peur de se montrer comme participant de plain-pied à la société... » (Corten, Dozon et Oro, éds 2003, p. 16). Son ambition, comme son nom l'indique, est d'être universelle. Pour ce faire, au cours de ses dix premières années d'existence, elle s'est efforcée de se doter d'une structure institutionnelle, de se développer à travers tout le Brésil, de se consolider économiquement, de marquer sa présence dans l'espace public à travers l'organisation d'un ample réseau médiatique (presse, radiophonie, télévision et informatique) et dans le champ politique. Une fois ces conditions réunies, l'Église universelle s'est lancée à la " conquête du monde », restant ainsi fidèle à la tradition religieuse dont elle est issue : le pentecôtisme. Elle a entamé son expansion internationale par le Paraguay, en 1985, les États-Unis, en 1986, le Portugal et l'Argentine, en 1989. Aujourd'hui un regard rétrospectif 
révèle que son expansion décolle vraiment à partir et tout au long des années 1990 et que celle-ci s'est produite quasi simultanément dans les pays d'Amérique, les pays africains de langue portugaise, en Afrique du Sud, au Portugal et, de là, dans les autres pays d'Europe et d'Asie, surtout au Japon et aux Philippines.

Aujourd'hui l'Église universelle est présente dans 80 pays du monde où elle compte près de 600000 fidèles. Elle existe dans toutes les Amériques à l'exception de Haïti, dans la moitié de l'Afrique; dans une vingtaine de pays européens et dans quelques pays d'Asie. Évidemment, dans la plupart des pays, sa présence est symbolique. En dehors du Brésil, les pays où l'Église universelle a plus de 50 temples sont l'Argentine, le Venezuela, le Portugal, la Côte d'Ivoire, le Mozambique, l'Afrique du Sud et les États-Unis.

Les spécialistes ont observé que la transnationalisation de l'Église universelle se fait par des individus pour lesquels elle peut être comprise, du point de vue linguistique et culturel, et là où elle parvient à développer son adaptation aux diversités locales, c'est-à-dire aux contextes culturels locaux (Freston 2003; Oro 2004). Son implantation dans les pays latino-américains du Cône sud débute en 1989. En 1994, elle avait déjà fondé 20 temples en Argentine, puis 46 en 1997 et 70 en 2001. Aujourd'hui, l'EURD en possède 86 et 47 lieux de culte appelés « annexes ». Ce n'est qu'en 1990 que l'Église universelle pénètre en Uruguay. Là, sa croissance est plus lente. En 2001, elle possédait 11 temples, alors qu'aujourd'hui on en compte 16 (6 à Montevideo et 10 dans l'intérieur du pays). Elle détient, par ailleurs, 29 « annexes » dans de petites villes de province.

\section{La légitimation politique}

L'Église universelle - en raison de ses propres caractéristiques - est, depuis sa fondation, une église controversée et polémique. C'est pourquoi, ces dernières années, au Brésil, aucune autre Église ou religion n'a, autant qu'elle, fait l'objet de reportages dans la presse à grands tirages, de thèses universitaires et de procès. Dans ce dernier cas, les accusations contre elle recouvrent un éventail considérable, allant du charlatanisme, de l'exercice illégal de la médecine (curandeirismo) et de l'escroquerie, jusqu'à l'implication dans le trafic de drogues et l'association de malfaiteurs, en passant par des irrégularités liées au droit du travail, des fraudes fiscales, des crimes financiers, des irrégularités comptables. Dans ce cas, comme l'a souligné Giumbelli (2002), c'est la légitimité de l'existence même de l'EURD en tant que religion qui est en cause.

Cependant, d'un point de vue juridique, l'Église universelle n'a jamais été condamnée, de manière définitive, pour ses pratiques religieuses, c'est-à-dire qu'elle n'a pas été juridiquement considérée comme criminelle pour les doctrines et les pratiques réalisées dans ses temples, pas même pour celles où se vérifient l'intolérance religieuse et la diffamation envers les religions afro-brésiliennes. Mais cela ne signifie pas pour autant qu'il en soit de même en ce qui concerne sa comptabilité, ni non plus qu'elle n'ait pas été contrainte de renoncer à plusieurs 
stratégies visant la conquête d'une plus grande légitimité au sein de la société brésilienne.

C'est seulement à partir de la seconde moitié des années 1990 que l'Église universelle et ses dirigeants ne font plus les gros titres des faits divers, qu'ils réussissent à réduire le nombre de procès et à acquérir une meilleure image sociale. Cela s'explique par la mise en œuvre d'une stratégie de consolidation en tant qu'institution, symbolisée par la construction d'immenses cathédrales dans les principales villes brésiliennes et par la diffusion, à travers ses propres médias, de l'image d'une religion sérieuse fournissant une assistance sociale. Mais elle a aussi démontré qu'elle détenait un important capital politique, se manifestant à chaque nouvelle élection, et qu'elle était capable de faire élire ses membres à d'importants postes de la politique brésilienne. En effet, l'Église universelle a débuté en politique en 1986, avec un député au Congrès national. À partir de là, elle a connu une progression constante : trois députés fédéraux et six députés des États sont élus en 1990 ; six députés au congrès national et huit aux assemblées législatives des États en 1994 ; dix-sept députés fédéraux et vingt-six députés des États en 1998 ; des dizaines de conseillers municipaux en 2000 et, aux dernières élections de 2002, seize députés fédéraux, dix-neuf députés des États et un sénateur de la République, élu à Rio de Janeiro avec plus de trois millions de voix. Il est important de souligner que tous les députés mentionnés sont des évêques et pasteurs de l'EURD et qu'ils représentent aujourd'hui, au sein des parlements des États, une force politique supérieure à celle de divers partis politiques. Il s'agit donc d'un " bloc » politique non négligeable dans la constitution des alliances. Et, bien évidemment, l'Église universelle a su tirer parti de son capital politique. D'ailleurs, c'est non seulement la compréhension de l'importance de l'arène politique en tant qu'espace permettant de calmer la vague des procès dont elle était la cible qui a motivé son entrée en politique, mais aussi, précisément, la conquête d'une plus grande respectabilité et reconnaissance sociales. Aussi paradoxal que cela puisse paraître, l'Église universelle semble avoir atteint son objectif, si l'on en croit le sondage réalisé par le respectable institut Data Folha et publié dans le journal Folha de São Paulo (4 janvier 2004) qui affirme qu'elle serait aujourd'hui perçue par la population brésilienne comme étant l'institution dont le pouvoir et l'influence ont le plus augmenté au Brésil entre 1995 et 2003.

\section{La confrontation entre l'Église universelle et les religions afro-brésiliennes}

Tout en naissant et en se consolidant en tant qu’Église entreprenante et insérée dans les médias et la politique, l'EURD s'est également montrée, depuis le début, agressive envers les autres religions qui se trouvent en concurrence avec elle pour l'enrôlement de fidèles. Cette agressivité est présente aussi bien dans les temples que dans les émissions médiatiques. Les années passant, cependant, elle a compris que la disqualification des "autres" se doit de respecter le degré de légitimité dont jouit chacune de ces religions au sein de la société brésilienne. 
C'est pourquoi on observe désormais une gradation de la relation conflictuelle de l'Église universelle avec les autres religions, qui tient compte non seulement de leur pouvoir politique, mais aussi des représentations sociales les concernant. Ainsi, aujourd'hui, le conflit opposant l'EURD à l'Église catholique est faible en raison de sa position historiquement hégémonique en Amérique latine et de son pouvoir politique encore important. De même, avec les Églises évangélistes, le conflit est limité, car l'EURD leur reconnaît une certaine légitimité religieuse. En revanche, il aurait tendance à se renforcer avec le mouvement du New Age et le spiritisme kardéciste, considérés tous deux comme des manifestations par lesquelles s'insinue le «mal satanique ». Ce conflit devient très virulent avec les religions afro-brésiliennes, envisagées comme des religions démoniaques et donc des voies privilégiées de l'action des démons responsables de tous les maux dont sont atteintes les personnes et la société.

C'est donc dans ce contexte de représentations symboliques que l'Église universelle va s'attribuer la mission de combattre Satan, présent dans les religions afro-brésiliennes, et de libérer ainsi les individus. Autrement dit, depuis sa fondation, elle a déclenché une "guerre spirituelle » sans trêve, radicale, contre les religions afro-brésiliennes, à tel point que ce combat «est devenu l'un des principaux piliers de sa doctrine » (Mariano 1995). La « lutte» se fait dans les temples à travers le rituel performatif de l'exorcisme, retransmis ultérieurement dans les émissions télévisées et radiophoniques. On y invoque les entités des religions afro-brésiliennes (aujourd'hui désignées par le terme générique de encostos) afin qu'elles "se manifestent », pour être ensuite humiliées et enfin exorcisées. Ce rituel, affirme Edir Macedo (1995, p. 36), fondateur de l'EURD, tient une « place centrale dans le culte ». C'est là que « les démons sont humiliés et même ridiculisés » (Macedo 1987, p. 134). Bien que les démons se manifestent presque quotidiennement dans les temples de l'EURD, c'est plus spécifiquement le mardi, lors des " séances spirituelles de décharge ${ }^{8}$ ", et le vendredi, dans les "réunions de libération ", que les rituels d'exorcisme sont les plus nombreux. Comme le rappelle Ronaldo de Almeida (2003), la libération finit par être structurellement associée, au sein de l'Église universelle, à un désensorcellement face aux sorts des religions afro-brésiliennes, dont la véracité est reconnue et acceptée. C'est aussi à travers ses médias, surtout par la radio et la télévision, que l'EURD véhicule sa doctrine selon laquelle le panthéon afro-brésilien est la cause principale de tous les maux et le contact avec ses fidèles l'une des principales formes de contagion par les forces maléfiques. Les stratégies de diffusion de cette doctrine se servent de montages de vidéo-clips, de témoignages et de reportages, abondamment illustrés par les symboles, les personnes et les images appartenant au champ religieux afro-brésilien. 


\section{Transnationalisation des religions brésiLiennes. II. Le cas de l'umbanda et du batuque en Uruguay et en Argentine}

Les religions afro-brésiliennes forment tout un éventail qui s'organise fondamentalement selon trois tendances : l'une africaine, avec le candomblé de Bahia, le xangô de Recife, la casa das minas du Maranhão et le batuque du Rio Grande do Sul ; ici les divinités sont les orixás et les vodums, et les symboles religieux ainsi que la langue rituelle sont africains, de tradition yoruba et éwé ; l'autre brésilienne, avec l'umbanda, aux influences spirites et catholiques très marquées ; là le panthéon est formé, entre autres, par les caboclos et les pretos-velhos ; enfin la troisième tendance qui croise des éléments symboliques des deux traditions précédentes, est incarnée par la quimbanda, qui rend un culte aux exus et pomba-giras ${ }^{9}$. L'umbanda et la quimbanda se sont aujourd'hui propagées dans tout le Brésil, tout comme le candomblé, la variante africaine la plus prestigieuse. Quant au batuque du Rio Grande do Sul, moins connu et moins étudié que les autres expressions afro-religieuses du Nord-Est, il est devenu la variante africaine la plus exportée vers les pays voisins du Cône sud. Avec l'umbanda et la quimbanda, ce sont les religions afro-brésiliennes qui se développent le plus en dehors de leur pays d'origine ${ }^{10}$.

\section{Les religions afro-brésiliennes en Uruguay et leur croissante légitimation sociale}

Les religions afro-brésiliennes ont commencé à se propager en Uruguay à la fin des années 1950, se développant rapidement pendant la dictature militaire, durant les années 1970 et jusqu'au début des années 1980 (Hugarte 1998). Il existe aujourd'hui de nombreux terreiros (maison de culte) d'umbanda et de batuque, surtout à Montevideo, mais ils essaiment également dans tout le pays. L'évaluation de la quantité de temples existants fait l'objet de discussions - Cisneros (1995) estime qu'il y en a un peu plus de 200. En revanche, personne ne conteste la quantité impressionnante de fidèles qui assistent tous les 2 février à la fête de Yemanjá sur la plage centrale de Ramirez. Depuis 1987 environ, la popularité de cette fête est telle que, selon Hugarte (1998, p. 44), « il n'y a actuellement aucun événement capable de réunir autant de monde que la fête de Yemanjá ». Face à ce pouvoir de rassemblement de plusieurs milliers de fidèles, intéressés et curieux, les journalistes, les fonctionnaires et les politiciens ont progressivement commencé à porter un autre regard sur ces religions.

Pour comprendre comment l'umbanda a acquis un degré supérieur de légitimité sociale en Uruguay - par comparaison avec l'Argentine -, il est nécessaire de prendre en compte les activités réalisées par deux groupes de leaders religieux qui, grâce à leur importante visibilité sociale, sont devenus des référents religieux face à la société globale. Ce sont eux qui, dans la quête d'une légitimation sociale pour leur religion ${ }^{11}$, ont le plus clairement développé des "cadres interprétatifs d'action collective " pour la construction d'une communauté religieuse. Pour ce 
faire, ils ont mobilisé des ressources économiques et culturelles internes et externes à leur religion et ont su tirer profit des opportunités offertes par le milieu social dans lequel elles se développent.

Le premier groupe de leaders religieux ayant monopolisé la représentation sociale de l'umbanda dans les années 1980, et ce pratiquement jusqu'à la fin des années 1990, a privilégié un « cadre d'action collective religieux » qui, progressivement, a glissé vers un cadre culturel. Les efforts engagés dans ce cadre (c'est-àdire selon cette façon de percevoir et d'organiser l'action collective) visaient principalement à rehausser le statut de l'umbanda/batuque en tant que religion et à obtenir l'union de ses pratiquants. Comme cela s'est produit en Argentine (et probablement sous son influence, étant donné les contacts fréquents entre les pratiquants des deux pays), l'accent a été mis sur les aspects culturels de la religion, son héritage africain et ses liens avec d'autres religions de la diaspora afro-américaine. Des «festivals» réunissant les membres des religions afrobrésiliennes (Hugarte 1998, p. 47) célébrés dans des hôtels, des salons de réception ou dans de petits stades, on est passé aux « congrès ", suivant le modèle universitaire, avec des exposés présentés par des leaders religieux et par des chercheurs en sciences sociales sensibles à leur cause ${ }^{12}$.

À partir de 1997 se produit une rupture dans la manière dont cette religion est représentée dans le pays. Un deuxième groupe de leaders religieux, plus jeunes et plus lettrés, va prendre une série d'initiatives importantes qui marqueront un tournant dans le type de relations établies entre la communauté afro-umbandista et la société globale uruguayenne. C'est la publication du journal Atabaque qui lui donnera une visibilité et un prestige social supérieurs. Ce mensuel de seize pages - actuellement dans sa septième année de vie - est le seul média umbandista qui a réussi à être publié régulièrement, sans interruption, et qui est en vente dans les kiosques. Dans les articles de ce journal, comme dans les déclarations publiques de ces leaders à d'autres médias, la revendication d'une pleine et entière liberté religieuse, ainsi que la suppression des normes législatives et des activités policières qui la restreignent, est devenue l'axe central de leur discours. On a justifié ces revendications par un discours mettant en avant : a) la quantité de fidèles de la religion dans le pays (citant toujours pour preuve la fête de Yemanjá), b) la qualité de citoyens uruguayens de ces derniers, c) la référence à la tradition nationale uruguayenne de respect des minorités et, surtout, d) l'énumération détaillée des droits constitutionnels et des lois qui protègent la liberté religieuse. On constate donc, dans les formes d'action collective de ce groupe, le passage d'un précédent cadre d'action collective culturel/religieux à un cadre civil, c'està-dire, de revendication de droits civils.

Les leaders, au lieu des formes de mobilisation incluant principalement la célébration de grandes fêtes religieuses ou de réunions dans des lieux publics fermés, vont désormais proposer aux fidèles afro-umbandistas des rassemblements ouverts dans des lieux stratégiques et à des points centraux de la ville, où ils 
arrivent à faire venir d'importants fonctionnaires d'État. Ils modifient et diversifient ainsi le type d'alliances établies avec d'autres secteurs et acteurs sociaux. Leur politique ressemble davantage à celle d'une $\mathrm{ONG}$ qu'à celle d'une fédération religieuse, des alliances étant nouées avec des secteurs et acteurs sociaux qui excèdent largement le religieux. Ils entretiennent ainsi des relations étroites avec le secteur le plus dynamique et populaire de la communauté noire, l'organisation Mundo Afro (Ferreira 2003), tout comme avec des organismes de défense des droits de l'homme et des organisations représentant d'autres minorités ethniques (juives ou indigènes).

\section{Les religions afro-brésiliennes en Argentine et leur problématisation croissante}

En Argentine, l'umbanda provient à la fois de Porto Alegre, où certains leaders argentins la découvrent dans les années 1960, et de Montevideo, à travers l'immigration de pères-de-saint uruguayens à cette même époque. La religion se développe lentement dans les années 1970, la pratique du batuque (connu aussi dans ce pays comme africanismo) venant s'ajouter à celle de l'umbanda à la fin de cette même décennie. À partir de 1983, avec le retour à la démocratie, les umbandistas/africanistas peuvent pratiquer plus librement leur religion et le nombre de temples augmente de façon remarquable. Au début des années 1990, quelque 450 temples avaient une existence légale, mais les pratiquants estimaient qu'il y avait au moins mille maisons de culte de religions afro-brésiliennes. La plupart des temples se situent dans le Gran Buenos Aires ${ }^{13}$ dans des zones habitées par les classes moyenne et moyenne-basse bien que, ces dernières années, ils aient été également installés dans la ville de Buenos Aires et dans des quartiers plus pauvres de la périphérie (Frigerio 2002b).

La situation actuelle de l'umbanda en Argentine est très différente de celle de l'Uruguay. Surtout parce que, contrairement à leurs pairs uruguayens qui ont conquis une certaine légitimité sociale, la stigmatisation des umbandistas argentins s'est accentuée au cours de ces dix dernières années. De plus, les relations entre l'État et les minorités ethniques et religieuses diffèrent notablement d'un pays à l'autre. Durant les deux dernières décennies, une douzaine de leaders umbandistas ont proposé des cadres d'action collective qui ont donné lieu à différents types d'initiatives publiques dans le dessein d'acquérir une plus grande légitimité sociale. Contrairement à l'Uruguay, où coexistent deux groupes de leaders avec des cadres différents (l'un culturel, l'autre de droits civils), en Argentine, ces cadres ont été proposés successivement - et finalement adoptés, non sans conflits. Dans le cadre religieux d'action collective ont été réalisés, dans les années 1980, plusieurs "congrès d'umbanda » où ont été reproduits des modèles issus du monde universitaire, comme la présentation d'exposés par les pères et mères-de-saint expliquant en détail les croyances, l'histoire de la religion et montrant les rituels. À travers ce genre d'activités organisées dans des locaux prestigieux du centre-ville, ils voulaient montrer que leur religion était une 
« vraie » religion, et dissiper ainsi les soupçons, circulant dans les médias et dans certains secteurs gouvernementaux, qui associaient uniquement l'umbanda à une secte magique dont l'activité principale était le sacrifice d'animaux.

Leurs activités ont réussi à améliorer l'image de l'umbanda auprès des fonctionnaires responsables des relations entre l'État et les religions non catholiques, mais ont connu des retombées médiatiques inégales. Certains journaux et revues se sont moqués des cérémonies publiques d'umbanda, réalisées lors de ces congrès. L'argumentation de quelques leaders religieux, préférant mettre en avant les aspects culturels de la religion, a fini par l'emporter et a abouti à la formation d'un nouveau cadre principal d'action collective. Deux événements, ayant secoué la communauté umbandista/africanista au début des années 1990, ont contribué à la diffusion et à la popularisation de ce nouveau cadre culturel d'action collective. Tout d'abord, la visite de l'Oni d'Ilé-Ifé (roi de la ville sainte des Yoruba) en Argentine et dans un terreiro d'umbanda en janvier 1990. Cette visite a permis de créer des liens entre l'ambassade du Nigeria et quelques leaders religieux, l'ambassade ayant commencé à soutenir les événements publics umbandistas, à travers la présence de son attaché culturel, ce qui n'était pas négligeable pour une religion stigmatisée. Le deuxième facteur a été le scandale médiatique qui a éclaté en juillet 1992, lorsqu'un prêtre catholique a accusé (injustement) un père-de-saint d'avoir rituellement sacrifié une petite fille. Au moment où les «sectes » étaient considérées comme un problème social, cette accusation a déclenché un vent de panique qui a gravement détérioré l'image de la religion, en sous-entendant que le sacrifice d'animaux pouvait conduire au sacrifice d'êtres humains (Frigerio et Oro 1998).

Conformément au nouveau cadre culturel d'action collective, on ne pense plus que la légitimation de la religion puisse venir de la mise en évidence des caractéristiques religieuses qui la rapprochent du catholicisme, mais plutôt de l'affirmation de son appartenance à une autre tradition culturelle et religieuse, la tradition africaine, millénaire, constituant l'une des racines de la culture latinoaméricaine, mais aussi de la culture argentine, bien que ce fait soit ignoré (Frigerio 2002b) ${ }^{14}$. À la fin des années 1990 , les actions collectives menées dans ce cadre avaient permis aux responsables religieux de se faire quelques alliés (universitaires, activistes culturels afro-américains, quelques rares diplomates), d'accéder à de prestigieux centres culturels et de gagner en partie la confiance des fonctionnaires du Secrétariat aux cultes. Cependant, cette amélioration de leur image ne s'est produite qu'au sein de cercles réduits : la légitimation sociale leur était encore refusée.

À la fin des années 1990 débute un processus de gestation d'un autre cadre d'action collective, insistant désormais sur l'action en terrain politique et sur les droits civils. Abandonnant les congrès dans des théâtres et des centres culturels, les africanistas/umbandistas ont alors organisé des réunions publiques dans des temples avec des politiciens auxquels ils ont voulu montrer leur pouvoir de 
rassemblement. En décembre 2002, alors que la campagne pour les élections présidentielles d'avril 2003 battait son plein, ils parvinrent à entrer en contact avec les candidats à la vice-présidence de deux des principales formations, auxquels ils remirent une pétition signalant six domaines dans lesquels ils sollicitaient la fin de la discrimination et de l'exclusion dont souffrait la communauté religieuse. Les actions collectives menées selon les cadres principaux antérieurs, soit les cadres religieux et culturels, n'ayant pas permis d'aller au-delà de simples revendications rhétoriques, dans des cercles réduits, en tant que "religion » ou «tradition culturelle » afro-américaine, les umbandistas formulèrent alors des revendications ponctuelles au nom de leurs droits en tant que citoyens.

À partir de 2003 environ, les afro-umbandistas des deux côtés du Rio de la Plata commencent à redouter un nouvel ennemi, auquel ils n'avaient prêté jusque-là que peu d'attention. Peut-être en raison de leur plus grande légitimité sociale en Uruguay qui leur permet de s'occuper de l'unique acteur social qui les prend encore pour cible privilégiée de ses attaques, ou bien comme moyen de justifier l'entrée en politique en Argentine, ou encore parce que la croissance de leur nouvel adversaire se fait de plus en plus visible, les pratiquants des religions afro-brésiliennes commencent à concentrer leur attention sur les offensives de l'Église universelle du royaume de Dieu. Les nouvelles qui arrivent de Porto Alegre, rapportant que certaines lois proposées par des législateurs évangélistes pourraient sévèrement limiter la réalisation de sacrifices d'animaux ou les célébrations religieuses avec les tambours, alimentent également cette nouvelle inquiétude. Commencée au Brésil, la guerre sainte se répand dans tout le Cône sud et les pères et mères-de-saint se mettent à reconsidérer les armes qu'ils ont à leur disposition pour y faire face.

\section{La guerre sainte au Brésil et dans les pays de la région du Plata}

\section{Église universelle versus religions afio-brésiliennes au Brésil}

Les religions médiumniques - le spiritisme, mais aussi l'umbanda et surtout le candomblé - ont été pendant longtemps victimes au Brésil de préjugés, de persécutions et d'attaques le plus souvent déclenchés par les autorités policières avec l'approbation de l'Église catholique et liés au discours raciste et ethnocentriste construit sur ces religions, surtout les afro-brésiliennes. Alors qu'on le croyait disparu, ce fantôme réapparaît sous la forme de ces " nouveaux ennemis beaucoup plus décidés et disposés à les expulser de la scène religieuse, des combattants qui font de la persécution des croyances afro-brésiliennes un acte de foi, aussi bien dans l'enceinte fermée et réduite des temples que dans l'espace illimité et public de la télévision et de la radio » (Prandi 2003, p. 23). Les Églises néo-pentecôtistes, et en particulier l'EURD, font de l'attaque et de l'agression symbolique envers le panthéon afro-brésilien - et par extension envers l'ensemble 
de ces religions - l'un des signes distinctifs de leur propre identité religieuse, ce qui signifie que, jusqu'à un certain point, elles ont besoin des religions afrobrésiliennes pour exister et se reproduire en tant qu'Églises.

Pendant les années 1980, les réactions des pratiquants des religions afrobrésiliennes aux agressions de l'EURD ont été peu nombreuses et espacées. Plusieurs facteurs peuvent expliquer cet état de fait. Premièrement, le caractère décentralisé et fragmenté de ces religions, dont l'organisation comprend des fédérations dans chaque État et une nébuleuse de terreiros, tous à la fois autonomes et rivaux entre eux, pris au milieu de quelques fragiles et petits réseaux d'alliances (Prandi 1991). Comme nous l'avons vu, le terme « religions afrobrésiliennes » cache une diversité de variantes régionales, à leur tour divisées en plusieurs « nations » africaines et différentes modalités plus syncrétiques et plus réafricanisées - ce qui fournit un spectre religieux très diversifié dans lequel les fidèles peuvent difficilement agir de façon uniforme. Même au sein de chacune des variantes régionales, dans un même État (le candomblé de Bahia ou le batuque du Rio Grande do Sul, par exemple), il est presque impossible de faire émerger une identité collective, un « nous » motivé par l'action commune - qui transcende les réseaux de parenté et de réciprocité rituelle (Frigerio 2003).

Deuxièmement, habitués depuis l'époque de l'esclavage à survivre aux persécutions des maîtres, à celles des gouvernements successifs des États et de la République fédérale brésilienne, ainsi qu'à celles de l'Église catholique, les pratiquants des religions afro-brésiliennes ont simplement considéré les attaques de I'EURD comme une offensive de plus. Comme le fait remarquer le père-de-saint Aristides Mascarenhas, directeur de la Federação Bahiana de Cultos AfroBrasileiros : "Nous avons déjà survécu à bien pire, et nous sommes encore là aujourd'hui » (Folha de São Paulo du 22 octobre 1995). Par ailleurs, l’Église universelle ayant été stigmatisée jusqu'au milieu des années 1990, ses attaques n'ont pas paru importantes, puisqu'elles provenaient d'un groupe religieux peu valorisé au sein de la société brésilienne. Cependant, plus l'EURD gagnait en respectabilité et en pouvoir politique, plus ses attaques devenaient inquiétantes et les réactions des pratiquants des religions afro-brésiliennes augmentaient. Les réponses furent variées et eurent lieu dans les États brésiliens les plus importants - ceux où l'on trouve également le plus d'individus qui se déclarent pratiquants de religions d'origine africaine (São Paulo, Rio de Janeiro, Bahia et Rio Grande do Sul).

Dans ce contexte, il s'avère particulièrement éclairant de séparer les initiatives des umbandistas, d'une manière générale, en deux groupes : d'un côté, celles qui ont fait appel aux tribunaux en portant plainte contre ces attaques; de l'autre, celles qui ont insisté, ou insistent, sur la nécessité pour les pratiquants des religions afro-brésiliennes d'entrer dans l'arène politique. Ceux qui proposent la première stratégie pensent que l'ordre juridique existant suffit à freiner les attaques de l'EURD, alors que ceux qui préfèrent la deuxième modalité estiment que 
c'est seulement par la participation politique que l'on peut influencer les centres décisionnels et endiguer les efforts des députés et conseillers municipaux de l'EURD, susceptibles de faire passer des lois qui limiteraient l'action des umbandistas - en particulier celles qui touchent à la protection des animaux et au contrôle des nuisances sonores en ville, pouvant compromettre la réalisation de cérémonies avec les tambours et les sacrifices rituels.

La préférence pour l'une ou l'autre de ces stratégies - qui ne s'excluent pas l'une l'autre - semble refléter le degré de légitimité acquis par les religions afro-brésiliennes dans chaque État. Nous examinerons ici deux exemples distincts, celui de Bahia dans le Nord-Est brésilien, et celui de Porto Alegre, dans l'État du Rio Grande do Sul, dans le sud du pays. Ces deux cas sont représentatifs non seulement des différentes stratégies employées et de la manière dont elles sont soutenues par d'autres acteurs sociaux, mais aussi en tant qu'ils mettent clairement en évidence les extrêmes entre lesquels oscille, au Brésil, la valorisation de l'héritage culturel, historique et génétique de la population noire. Bahia a fait de sa population noire un élément central de son identité, alors que dans le Rio Grande, au contraire, prime son invisibilité. Dans la construction de l'identité gaícha, c'est à la fois l'héritage et la présence actuelle de la communauté afroriograndense que l'on a sous-estimés (Oliven 1992). On n'y reconnaît pas non plus la popularité inédite du batuque qui, naissant parmi la population noire de l'État, est aujourd'hui pratiqué par des individus de toutes origines ethniques (Oro 1989).

C'est dans l'État de Bahia, là où la religion et l'héritage noirs sont le plus valorisés, que les pais-de-santo ont considéré qu'ils étaient suffisamment protégés par l'ordre juridique existant pour pouvoir dénoncer la puissante EURD dans les tribunaux. En janvier 2003, par exemple, huit pères-de-saint ont entrepris des actions en justice contre cette Église, exigeant un droit de réponse dans l'émission Ponto de Luz, car des symboles et des éléments du candomblé y étaient utilisés pour critiquer les religions afro-brésiliennes ${ }^{15}$. Les pères-de-saint ont également demandé - en plus d'un droit de réponse - que les responsables du programme soient punis.

Dans ce même État, un an plus tard, les pratiquants des religions afrobrésiliennes obtiennent leur première victoire juridique face à l'EURD. En janvier 2004, un juge civil de Salvador a prononcé une sentence qui obligeait l'EURD à indemniser les membres de la famille d'une mère-de-saint, à hauteur de près de 400000 dollars, pour préjudice moral, en raison d'une photo d'elle indûment publiée dans le journal de l'Église, la Folha Universal, pour illustrer un article intitulé « macumbeiros charlatans portent atteinte à la bourse et à la vie des clients ». Trois mois après la diffusion de l'article, cette mãe est morte de tristesse. Selon le journaliste qui a rapporté les faits dans un journal local, la décision « est considérée comme un pas important dans la lutte du culte africain contre l'intolérance religieuse dont le candomblé et l'umbanda sont victimes ces dernières 
années, même à Bahia connue pour être le berceau de la culture noire au Brésil 》 (A Tarde, Salvador; du 20 mars 2004. Italique des auteurs). Bien que l'affaire ne soit pas encore close, il est significatif que ce soit dans l'État considéré comme le berceau de la culture noire au Brésil que, pour la première fois, les religions afro-brésiliennes aient remporté une victoire sur l'EURD dans une cour de justice. À la requête d'un conseiller de la municipalité de Salvador, le jour de la mort de la mère-de-saint en question a été déclaré «journée de lutte contre l'intolérance religieuse ». Ce soutien apporté aux religions afro-brésiliennes, face aux offensives de l'EURD, par des fonctionnaires et législateurs de l'État de Bahia - dont on ne trouve pas d'équivalent dans d'autres États où ces religions ne sont pas considérées comme un élément central de la culture régionale - s'est manifesté à d'autres reprises.

En avril 2003, un évêque et deux pasteurs de l'EURD ont été dénoncés, auprès de la justice de Bahia, pour discrimination religieuse par le procureur de Justice et Citoyenneté du ministère public d'État. Selon le journaliste qui a suivi l'affaire, " les accusés se sont attaqués plusieurs fois au candomblé dans le programme Ponto de Luz, diffusé par la TV de Salvador [où] les évangélistes présentent une prétendue ex-mère-de-saint aflirmant avoir fait un "travail" de macumba [magie noire] pour tuer un rival et ce dernier, selon elle, serait mort ». De son côté, le procureur a affirmé que « la liberté de culte est garantie par la Constitution et que la religion afro-brésilienne est protégée par la constitution de l'État " (Reportage "L'Église universelle est accusée de discrimination religieuse », Estado de São Paulo, du 16 avril 2003. Italique des auteurs). À la même époque, plusieurs groupes de législateurs bahianais se sont également intéressés à la question : en mars s'est tenue, dans la chambre des conseillers municipaux de Salvador, une séance spéciale sur le thème de l'intolérance religieuse et, en mai, la commission spéciale pour les affaires de la communauté afro-descendante de l'Assemblée législative de Bahia a organisé une audience publique dans un terreiro de candomblé pour discuter de la discrimination religieuse. C'est également suite aux efforts entrepris par un député bahianais que, pour la première fois, le Congrès national a abordé à Brasilia le thème de la religiosité afro-brésilienne - y compris celui des agressions médiatiques dont elle était victime - dans la séance « Religions afro-brésiliennes : tradition et résistance ». Celle-ci n'était pas seulement présidée et organisée par le député fédéral de l'État de Bahia, mais comptait également sur la participation du " Mouvement contre l'intolérance religieuse », une organisation dont le siège est à Salvador et qui est composée par des individus appartenant à divers groupes religieux. Plusieurs fonctionnaires bahianais (du gouvernement et des municipalités) étaient présents. Le sous-titre de cette séance, " tradition et résistance », montre que ces religions sont valorisées en tant qu'exemples de résistance culturelle de la part d'un groupe ethnique déterminé et non pas en tant que patrimoine spirituel commun à tous les Brésiliens, indépendamment de leur couleur de peau. 
Afin d'évaluer l'influence des constructions narratives dominantes - dans le cas présent, celles de la région - sur les stratégies que peuvent développer les pratiquants des religions afro-brésiliennes face aux offensives de l'EURD, prenons maintenant en considération le cas du Rio Grande do Sul, le deuxième État plus « blanc » du pays, où ces religions existent depuis plus d'un siècle (Oro 1989). À l'intérieur de cet État, il y a près de 40000 terreiros et, pourtant, il semblerait qu'il n'y ait de réactions d'aucune sorte, de la part des fédérations ou des membres des religions afro, contre l'intolérance religieuse, et ce, au moins jusqu'en 2002. Cette année-là, à l'occasion de la « XI ${ }^{\mathrm{e}}$ semaine de la conscience noire de Porto Alegre ", une commission pour la défense des religions afro-brésiliennes a été formée pour s'opposer à la virulence des attaques de l'EURD. Cette commission était constituée par des leaders religieux, intellectuels noirs et militants du mouvement noir du Rio Grande do Sul, et présidée par une mère-de-saint (noire) de Porto Alegre. La commission de défense des religions afro-brésiliennes a réalisé trois séminaires importants : deux dans l'État et un autre au niveau national, en mars 2004. Après presque deux ans de réunions et d'activités, cette commission, en intégrant également les décisions prises dans les séminaires déjà cités, réfléchit à la mise en œuvre d'actions plus concrètes contre l'intolérance religieuse :

a) la nécessaire union des efforts entre les terreiros, les fédérations et le mouvement noir. Reste à savoir si cette tentative sera couronnée de succès, étant donné les disputes déjà déclarées entre les divers acteurs sociaux impliqués dans le processus ;

b) l'engagement d'une action en justice contre l'EURD, qui constitue pour le moment une possibilité car, d'une part, on estime nécessaire d'acquérir d'abord une plus grande légitimité sociale, pouvant se répercuter également dans le domaine juridique et, d'autre part, on reconnaît ne pas avoir, dans ce domaine, de spécialistes capables d'intenter un procès bien argumenté, alors que l'EURD dispose d'un groupe d'avocats compétents et d'une profonde maîtrise du champ juridique lui garantissant la victoire dans les tribunaux. Face à cela, la commission propose de développer son action juridique, avec l'aide d'un avocat du mouvement noir qui participe aux réunions depuis quelques mois, pour pouvoir, dans l'avenir; engager des poursuites judiciaires, mais «cette fois-ci pour gagner ». Ainsi, la condamnation de l'EURD à Bahia, mentionnée plus haut, a motivé les membres de la commission à faire progresser l'idée d'une future action en justice contre l'EURD ;

c) la possibilité de créer un local qui serait le siège de divulgation de la cause afro-religieuse, comprenant une librairie, une maison d'édition et une chaîne de radio, puisqu'on reconnaît que la force de l'EURD aujourd'hui repose également sur son importante machine médiatique. La commission intervient aussi dans les écoles, en donnant aux élèves des cours de formation et d'orientation sur les religions afro-brésiliennes, proposant également de lutter pour la création d'un 
enseignement sur la théologie africaine à l'université, principalement dans l'État du Rio Grande do Sul, et prônant l'instauration dans cet État d'une journée pour la défense des « religions africaines ».

Mais, aujourd'hui, l'accent est mis surtout sur le domaine politique en tant que champ d'action possible, partant, encore une fois, de la lecture faite par les membres de la commission, selon laquelle le pouvoir de l'Église universelle provient, en grande partie, de sa forte présence sur la scène politique, dans les chambres municipales, dans les assemblées législatives et au Congrès national. C'est pourquoi, pour la commission, la politique est le théâtre d'action le plus crédible contre l'intolérance religieuse. De ce fait, il devient nécessaire pour la religion afro d'avoir également ses représentants politiques, afin de faire pression et d'agir contre l'intolérance religieuse dont elle est victime. Ainsi, en vue des élections pour les postes de conseillers municipaux et de maires en octobre 2004, la commission était en train d'identifier des personnes susceptibles de se présenter aux postes législatifs municipaux des principales villes de l'État. Cependant, un doute important, qui n'avait pas encore été résolu au moment de l'écriture de cet article, subsistait : savoir si l'on désignait et soutenait des personnes de religion afro-brésilienne (de préférence des pères et mères-de-saint) ou bien seulement des sympathisants. Dans ce sens, deux politiciens ont développé leurs contacts avec la commission, afin d'obtenir son soutien, en se montrant intéressés à défendre la cause des Afro-descendants et des adeptes des religions afro-brésiliennes; la commission pencha alors pour le soutien de ces candidats non religieux.

Cet exemple révèle, tout d'abord, la séparation existant dans cet État, dans un premier temps, entre négritude et religion. Selon le témoignage de la présidente de la commission, en onze ans de célébrations successives de la «Semaine de la conscience noire », on n'a jamais intégré de leaders des religions afro-brésiliennes au mouvement de revendication de la culture noire dans cet État, les revendications ethniques et religieuses étant séparées. C'est pourquoi un groupe de pères et mères-de-saint propose, aujourd'hui, un cadre culturel d'action collective : ils pensent en effet que c'est en participant au mouvement social, qui cherche à revendiquer la présence et la culture noires dans un État traditionnellement considéré comme blanc, qu'ils réussiront à obtenir des appuis pour leur religion. Ils doivent d'abord revendiquer la présence noire au sein de la construction narrative dominante du Rio Grande do Sul, pour pouvoir ensuite engager d'autres actions, cette fois-ci pour la défense de leur religion. Les militants noirs sont, pour le moment, leurs meilleurs alliés et, contrairement à ce qui s'est passé à Bahia, ils n'ont reçu que peu ou pas de soutien de la part des autres religions.

Progressivement - peut-être en raison des attaques de législateurs évangélistes ayant presque réussi à faire passer des lois qui auraient limité le libre exercice de leur religion - les mères et pères-de-saint envisagent de mener leur action à l'intérieur d'un cadre politique, appelant leurs fidèles à voter pour des candidats 
appartenant à, ou soutenant, cette religion. Les leaders des fédérations d'umbanda et de batuque, quant à eux, ont oscillé entre des cadres d'action collective religieux et politiques. Dans le premier cas, ils essayaient de valoriser leur religion en organisant des réunions dans de prestigieuses institutions où les pères-de-saint et les chercheurs en sciences sociales abordaient les différents aspects des croyances afro-brésiliennes. Dans le second cas, quelques présidents de fédérations ou leaders de groupes de terreiros, unis par leurs liens de parenté rituelle, se sont présentés aux élections de conseiller municipal et de député, obtenant toutefois de très mauvais scores.

Bien qu'ils les envisagent comme une possibilité pour l'avenir, ils n'ont pas encore eu recours à des actions en justice, car ils craignent de ne pas être assez convaincants. Cela montre comment la construction narrative dominante de cet État, minimisant ou niant les apports noirs dans le Rio Grande do Sul, fait des pratiquants des religions afro-brésiliennes des outsiders, des pratiquants de religions de seconde zone, qui doivent éduquer leurs concitoyens sur la présence et l'héritage noirs dans leur culture, avant de pouvoir revendiquer leurs droits religieux dans les tribunaux. De même, ils se doivent d'entrer en politique pour obtenir des représentants umbandistas ou batuqueros qui les défendent face aux législateurs de l'EURD. La possibilité de défendre leurs droits de citoyens, conformément aux lois en vigueur qui pénalisent la discrimination religieuse, semble lointaine et improbable dans un contexte où ils sont malmenés par des représentants de l'État et, notamment, par la justice et la police. La possibilité et la volonté, dont ont fait preuve les pères-de-saint bahianais, d'avoir recours avec succès aux tribunaux, de compter sur l'appui de législateurs, de fonctionnaires et de journalistes face aux attaques de l'EURD, ne sont pas partagées par leurs frères religieux de Porto Alegre, dans un État traditionnellement orgueilleux d'une forte présence d'immigrés européens. Nous verrons que les religions afro-brésiliennes en Argentine et en Uruguay sont confrontées à un dilemme semblable.

\section{EURD versus umbanda en Uruguay}

Depuis plusieurs années, les divers groupes évangélistes, devenus populaires à Montevideo, ont choisi les umbandistas pour ennemi numéro un. La popularité conquise par la fête de Yemanjá, comme nous l'avons vu, y a sans doute contribué car, en rassemblant des milliers de fidèles sur la plage la plus centrale de la ville, les religions afro-brésiliennes ont gagné en visibilité, ce qui a stimulé la concurrence pour capter une clientèle religieuse ayant des caractéristiques similaires. C'est pourquoi l'intensité du conflit est plus forte, ou du moins plus manifeste, qu'en Argentine.

Dans leurs articles sur l'expansion des Églises pentecôtistes brésiliennes en Uruguay, Hugarte (1992) et Guigou (1993) signalent que, depuis son arrivée en 1986, l’Église « Dieu est Amour » (Iglesia Dios es Amor) était celle qui avait le 
plus de succès et de visibilité. Selon Guigou (1993, p. 3), le conflit avec l'umbanda se déroulait principalement sur trois fronts : 1) l'espace rituel pentecôtiste, à travers les exorcismes, 2) l'espace public, surtout pendant la fête de Yemanjá et 3) l'espace radiophonique pentecôtiste. L'EURD est à peine mentionnée dans ces études, étant donné son faible développement à l'époque ;il est seulement fait référence au rachat, par l'EURD, du premier local occupé par " Dieu est Amour », après que celle-ci ait déménagé dans un cinéma sur l'avenue centrale « 18 de Julio ".

À la fin des années 1990, les attaques surtout radiodiffusées de l'Église Dieu est Amour (Hugarte 1998, pp. 20-21) sont remplacées par celles de l'Église universelle du royaume de Dieu lancées dans ses émissions télévisées, diffusées en fin de programmation sur les chaînes les plus importantes, ajoutant ainsi à la dispute une arène nouvelle et plus visible. Ce changement reflète également le sort des deux Églises néo-pentecôtistes; à partir du nouveau millénaire, l'EURD se substitue à « Dieu est Amour » en tant que principale Église néo-pentecôtiste brésilienne à Montevideo et son développement dans le pays commence à s'accélérer. Ainsi, en décembre 2003, l'EURD ne possédait des locaux, propriétés ou locations, que dans 7 départements uruguayens (sur 19 au total), alors qu'en avril 2004 elle était déjà parvenue à en avoir sur la quasi-totalité du territoire national (soit dans 16 départements). En juin 2004, elle possédait 16 temples en Uruguay (6 à Montevideo et 10 dans l'intérieur du pays) et 28 annexes. Bien que ces annexes soient des locaux loués, parfois seulement pour quelques heures par semaine, il est significatif de constater que, six mois auparavant, elle n'en possédait que huit ${ }^{16}$.

Les attaques proférées par l'EURD contre l'umbanda sur les écrans télévisés n'ont pas, dans un premier temps - comme cela s'est produit au Brésil -, inquiété outre mesure les umbandistas, davantage préoccupés par leur légitimation aux yeux d'acteurs sociaux plus importants. Le premier groupe de leaders n'y a pas prêté une attention particulière. Le deuxième, réuni autour du journal Atabaque, a d'abord considéré ces attaques comme un front de combat de plus parmi ceux qui s'étaient déjà déclarés, se limitant à demander aux chaînes de retransmission un droit de réponse. Cependant, au fur et à mesure que grandissait leur légitimité face aux fonctionnaires et législateurs uruguayens, ils ont commencé à considérer comme inacceptables les agressions télévisées quotidiennes de l'EURD.

En mars 2003, l'affaire a fait l'objet d'un éditorial dans l'Atabaque, dans lequel on peut saisir le cœur de l'argumentation que ce groupe utilisera dorénavant pour fustiger les offenses de l'Église universelle et discréditer publiquement cette Église. Dans le texte, ils exhortaient la chaîne qui transmettait le programme de l'EURD à :

[...] contrôler ce qui se passe avec les émissions de ladite Église universelle, à plus forte raison sur une chaîne qui se vante, dans son slogan, d'être " uruguayenne ». Ces émissions faites par des étrangers intolérants qui vont à l'encontre de tout ce que 
représente notre pays, en terme d'origine et de diversité culturelles, composantes de notre réalité sociale, malmènent la tradition de respect des différences, revendiquée par l'Uruguay. Ces Brésiliens [...] vont jusqu'à s'opposer avec arrogance aux intérêts économiques nationaux puisqu'il est bien connu que le tourisme est l'une des principales sources de devise dans le pays et que la fète de Yemanjá représente l'une des plus grandes attractions touristiques estivales. [...] L'attitude agressive et discriminante des membres de Pare de Sufrir n'est pas non plus conforme aux lois en vigueur dans notre législation, qui défendent l'égalité, la fraternité et la tolérance entre les personnes... Les umbandistas uruguayens, que l'on compte par centaines de milliers sur les plages le 2 février, ne regardent plus aujourd'hui la chaîne 10 car ils se sentent gravement offensés. (Atabaque, 65, mars 2003, p. 2. Italique des auteurs)

Cette argumentation suit le cadre interprétatif de défense des droits civils, utilisé par ce groupe d'afro-umbandistas, puisqu'elle met l'accent sur la structure juridique qui les protège et à laquelle les agressions de l'EURD porteraient atteinte (dans des textes ultérieurs, ils diront explicitement qu'elles violent l'article 5 de la Constitution nationale). Elle souligne également la présence effective des religions dans la société uruguayenne, rendue évidente grâce à la fète de Yemanjá, et elle insiste particulièrement sur l'identité uruguayenne de ses pratiquants, par contraste avec l'identité étrangère des pasteurs brésiliens, méprisant la tradition de tolérance propre à l'Uruguay. Cette base argumentative sera maintenue et approfondie au fur et à mesure qu'elle sera exposée, non seulement dans les pages de leur journal, mais aussi dans les missives adressées à la Cour suprême ou à l'organisme d'État responsable des chaînes hertziennes.

En juillet 2003, le conflit a pris une dimension transnationale. Le journal Atabaque, datant de ce mois, a rapporté plusieurs informations sur le conflit existant dans plusieurs localités brésiliennes mais aussi argentines. Le saut qualitatif dans la préoccupation vis-à-vis des attaques de l'EURD était dû à la vive inquiétude provoquée à Porto Alegre par l'adoption d'une loi, présentée par un législateur évangéliste, censée limiter les sacrifices d'animaux. Cette nouvelle n'a pas été seulement transmise via des contacts personnels - les pais uruguayens ayant des contacts réguliers avec leurs pairs brésiliens -, mais a aussi largement et rapidement circulé sur internet, à travers des chaînes de mails et des forums de discussion sur les religions afro-brésiliennes.

En août 2003, on a appelé à une mobilisation publique face au Palais de Justice, dans les pages d'Atabaque ainsi qu'à travers des chaînes de mails, afin de défendre la religion contre cette nouvelle et inquiétante menace. La situation d'autres pays, et particulièrement celle du Brésil offrant un scénario à éviter, a été invoquée pour justifier le rassemblement qui, s'il a été moins suivi que prévu, a néanmoins su attirer l'attention des médias sur le problème qui accablait les umbandistas. Suite à cette rencontre, une pétition a été présentée à la Cour suprême, réunissant deux mille signatures. L'action a été suivie par la présentation d'une Action de protection auprès d'un tribunal de justice civile. Aucune de 
ces initiatives n'a suscité une réponse favorable de la part de l'État, puisqu'elles ont été ignorées et rejetées par les institutions auxquelles elles avaient été adressées, les afro-umbandistas ayant fait appel par la suite. Peu de temps après, ils ont également envoyé une lettre à l'URSEC (Unité régulatrice des services de communication), l'organisme national chargé de contrôler l'usage des ondes hertziennes.

Début 2004, deux nouveaux événements ont encore avivé les craintes des afro-umbandistas. En janvier, les journaux ont annoncé que des membres de l'EURD avaient formé un " Mouvement d'action chrétienne », qui soutiendrait l'une des factions internes du Parti Colorado, annonçant ainsi l'entrée de cette Église dans la politique uruguayenne ${ }^{17}$. En février; le jour de la fête de Yemanjá, des graflitis agressifs ont été découverts sur le monument à cet orixá, ainsi que dans deux santerías, lieux de vente de produits de la religión (terme qui désigne, dans le Cône sud, comme à Cuba, les différentes modalités de culte d'origine africaine). La phrase offensive, répétée en toute occasion, était la suivante : "Yemanjá, la reine des mensonges ». Les afro-umbandistas, réunis autour d'Atabaque, ont vu dans cet épisode une conséquence directe des prêches agressifs télévisés des pasteurs de l'EURD.

Par peur du pouvoir que l'EURD pourrait parvenir à acquérir en Uruguay, si son actuel pouvoir économique se voyait renforcé par sa participation à la politique du pays, les directeurs d'Atabaque ont non seulement décidé de poursuivre leurs revendications auprès des institutions et organismes d'État, mais ont également jugé nécessaire de livrer bataille dans le même champ que leurs concurrents : la politique partisane. Voici, dans une note parue dans Atabaque, les fondements sur lesquels reposait cette décision :

[Les attaques de l'EURD en Uruguay et au Brésil] nous obligent impérativement à tracer un chemin politique pour les umbandistas. Si nous nous désistons, ils introduiront les leurs au Parlement et dans les intendances et, de là, ils mettront à exécution leur mission d'extermination de l'umbanda. Nous devons être présents dans les centres de décision pour pouvoir protéger nos cultes face à ces fondamentalistes de la foi. C'est la seule manière de faire au moins entendre la voix de notre religion à l'heure de légiférer. Ce qui est arrivé à nos frères brésiliens est un exemple clair de ce que nous ne devons pas reproduire. [...] Étant donné le contexte actuel, c'est la seule issue possible, réalisable à partir des centres de pouvoir auxquels on accède via la politique. ("La Iglesia Universal y su repudio hacia los afro-umbandistas : no cesan los ataques en Uruguay ", Atabaque, 76, février 2004, p. $11^{18}$ )

Peu après, le journal Atabaque demande à être intégré - et est accepté - à l'Encuentro Progresista-Frente Amplio (groupement de centre gauche qui a remporté en 2004 les élections). De cette façon, un groupe d'umbandistas participe explicitement à une formation politique, bien qu'ils ne prétendent pas, comme ils le précisent dans leur journal, parler au nom de tous les pratiquants de la religion. Dans les jours suivant leur entrée en politique, il a été clairement signalé à ce 
groupe d'afro-umbandistas qu'il leur faudrait payer le prix pour ce choix stratégique. Les coûts de leur affiliation politique ne font en effet aucun doute dans ce message, qui leur a été envoyé par un conseiller de l'un des députés du Parti Colorado qui considérait les avoir aidés quelques années auparavant :

[...] vous aviez une légitimité - que vous avez désormais perdue - pour traiter avec tous les partis politiques et pour que ceux-ci s'intéressent à vos revendications. Désormais, vous ne devrez compter que sur une seule force politique, et nous-mêmes - n'ayez pas le moindre doute là-dessus - nous ferons notre possible pour que vous n'ayez aucun succès, ni politiquement ni religieusement. (Message rendu public par les directeurs d'Atabaque et partiellement reproduit dans le journal La República, " Denuncias de los afro-umbandistas », du 30 avril 2004)

En avril 2004, après d'infructueuses initiatives auprès de la Cour suprême de justice, d'un tribunal civil, d'un tribunal pénal et de l'URSEC, ils ont été reçus par la commission des Droits de l'Homme du Parlement, à laquelle ils ont présenté leurs plaintes, ainsi qu'une vidéo de compilations des émissions Pare de Sufrir où l'on s'attaque à leur religion.

\section{EURD versus umbanda en Argentine}

Les umbandistas/africanistas argentins se sont montrés moins préoccupés que leurs collègues uruguayens par les attaques de l'EURD, très certainement parce que, traditionnellement, ils ont dû faire face aux critiques et offensives d'acteurs sociaux plus prestigieux et plus visibles au sein de la société argentine. Pendant les années 1980, ils ont d'abord dû lutter contre la méfiance des fonctionnaires du Secrétariat aux cultes, puis contre celle des journalistes et de leurs plus tenaces critiques d'alors, à savoir les sociétés protectrices des animaux. Au début des années 1990, alors qu'ils étaient au centre d'une vague de panique, ils ont affronté les accusations d'un prêtre catholique, qui les qualifiait d'assassins, et celles des membres de groupes anti-sectes, les considérant désormais comme une " secte dangereuse », imputations formulées dans toutes sortes d'émissions télévisées et à tous les horaires. Une fois passée cette vague de panique, dans la deuxième moitié des années 1990, ils se sont trouvés face à des dénonciations d'un groupe de prétendus pères-de-saint, convertis au pentecôtisme, les accusant de faire le mal et d'abuser de la confiance des gens. Bien que ce groupe n'ait pas participé à d'importants programmes journalistiques ou d'intérêt général - comme ce fut le cas des experts anti-sectes dans les années précédentes -, il est cependant parvenu à un certain degré de visibilité dans des talk-shows programmés l'après-midi, sur des chaînes hertziennes. Cette visibilité et le fait que ces individus revendiquent un passé de pères et mères-de-saint ont particulièrement exaspéré les umbandistas/africanistas locaux.

Dans ce contexte, les attaques de l'EURD, une Église stigmatisée et sans visibilité en Argentine, lancées dans des émissions nocturnes, sur des chaînes 
câblées ou après la clôture de la programmation sur une chaîne hertzienne, ne représentaient pas un danger comparable aux adversaires précédents. Par ailleurs, le mode sur lequel étaient faites les critiques de l'EURD les rendait sans doute moins offensantes que les agressions dont ils avaient souffert de la part d'acteurs sociaux plus importants. Dans les émissions Pare de Sufrir de l'EURD, observées en 2003 et 2004, étaient présentés quotidiennement deux témoignages de personnes sauvées par l'Église après avoir eu contact avec des « esprits ». L'une était toujours un individu ayant fréquenté des temples de religions afrobrésiliennes ; l'autre avait consulté des guérisseurs et des devins très différents. Le mot "umbanda » n'a presque jamais été mentionné dans ces émissions, qui mettaient en avant des expressions comme « temple des esprits » ou " servir les esprits ». En revanche, les mots « travaux » ou " despachos » ${ }^{19}$ étaient eux utilisés, termes considérés comme appartenant à leur univers discursif par ceux qui avaient fréquenté les religions afro-brésiliennes. Les termes " pai » ou " mãe », dont la signification est claire même pour un public profane, n'étaient presque pas utilisés, ou seulement de façon exceptionnelle, par quelques témoins. La référence la plus directe aux religions afro-brésiliennes intervenait lors de reportages intitulés "Manières d'entrer en contact avec les esprits", dans lesquels on montrait, au son des tambours, des images de cérémonies d'umbanda (avec possessions et danses), d'offrandes ou de statuettes d'Exu, plus facilement reconnaissables par les profanes. Cependant, les accusations n'ont jamais été plus explicites que dans les émissions séculières de la télévision hertzienne, lorsqu'elles étaient proférées par quelques prêtres catholiques, « experts en sectes », ou par les apostats convertis aux religions évangélistes.

Comme en Uruguay, les umbandistas/africanistas argentins ont commencé à s'inquiéter davantage des actions de l'EURD à partir du moment où a été annoncé le possible soutien de cette Église à l'un des candidats aux élections présidentielles argentines de 2003. Il est vraisemblable, toutefois, que cette situation n'ait été qu'un prétexte pour justifier le soutien politique des umbandistas à l'un des candidats. Les mauvaises relations de certains leaders religieux avec le directeur du Registre national des cultes, les diffĩcultés croissantes rencontrées par les umbandistas pour s'y faire inscrire (Frigerio et Wynarczyk 2004) et les nombreuses annulations d'inscription dont ils étaient victimes... bref, la détérioration du bon rapport qu'ils avaient réussi à établir avec les fonctionnaires de la section des cultes a été probablement la raison principale qui les a poussés à trouver d'autres interlocuteurs face à l'État.

Comme en Uruguay, la situation brésilienne actuelle a été donnée en exemple pour illustrer la menace potentielle de la progression politique de l'EURD en Argentine. Dans le seul journal umbandista existant début 2003, publié par le Front qui était censé les rassembler; on signalait, pour justifier leur entrée en politique, que : 
D'autres confessions religieuses ont fait la même chose. L'Église universelle s'est par exemple rapprochée du candidat Carlos Menem, réclamant une nouvelle loi des cultes et aurait, paraitt-il, offert des fonds pour la campagne [...]. Les membres du Front soulignent la menace que représente l'Église universelle pour la liberté de culte. On retrouvera certainement dans son projet de loi des cultes la même intolérance manifeste que celle qu'ils aftīchent dans leurs médias. Même au Brésil, on trouve des précédents de lois anti-umbandistas/africanistas [...]. La menace n'est pas surévaluée, il est évident que, lorsqu'elle aura obtenu une représentation politique, l'Église universelle fera la même chose en Argentine. Les leaders du Front espèrent d'ici là avoir formé un réseau capable de contenir la discrimination qui sévit dans de nombreux secteurs de la société. (Janvier 2003. Italique des auteurs)

\section{Conclusion}

La faiblesse ou l'absence de réponse aux attaques de l'EURD de la part des pratiquants de religions afro-brésiliennes, constatée par Oro (1997) il y a quelques années, semble désormais appartenir au passé. Ces dernières années, dans tout le Brésil, mais aussi en Uruguay et en Argentine, les pratiquants de religions afro-brésiliennes ont essayé - avec plus ou moins d'intensité - une série de stratégies, notamment judiciaires et politiques, pour freiner ces attaques. Malgré la légitimité accrue acquise par les pratiquants de religions afro-brésiliennes ces dernières années, il est clair qu'il reste un long chemin à parcourir pour qu'ils soient reconnus comme des sujets de plein droit. Bien que l'EURD ait dernièrement utilisé plusieurs pistes pour déguiser ses attaques - en évitant, notamment, de mentionner dans ses programmes télévisés les mots " umbanda ", " candomblé » ou des noms spécifiques se rapportant à leurs entités spirituelles -, l'usage d'euphémismes comme " mãe de encosto », " maison des esprits » et, surtout, les images utilisées dans les reportages sur les façons d'entrer en contact avec les esprits malins ou les fictions racontant les cas d'individus affectés laissent peu de doute sur la religion désignée comme la cause de tous les maux. Les autorités nationales, législatives et judiciaires, des trois pays concernés ont, dans l'ensemble, ignoré le conflit ou bien, lorsqu'elles devaient s'y référer, ont privilégié une interprétation du droit sur la libre expression religieuse, révélant le caractère minoritaire et quelque peu marginal des religions afro-brésiliennes. En effet, si les allusions au caractère démoniaque de certaines pratiques et croyances religieuses - faites de manière indirecte mais qui restent identifiables - avaient été dirigées, non plus contre l'umbanda, mais contre une religion majoritaire comme le catholicisme ou contre une religion minoritaire jouissant d'une légitimité et d'un pouvoir social supérieurs comme le judaïsme, les lois contre la discrimination religieuse en vigueur dans les trois pays auraient probablement été activées pour éliminer ou tempérer les attaques de l'Église universelle.

L'importance du degré de légitimité sociale d'une religion déterminée, pour l'obtention du soutien de l'État à ses revendications, apparaît clairement dans le 
fait que c'est, pour le cas brésilien, justement à Bahia que les luttes contre l'Église universelle au tribunal ont le mieux réussi. C'est dans cet État que les religions afro-brésiliennes jouissent du plus grand prestige social puisqu'on les considère comme partie intégrante de l'héritage culturel noir, tenu pour un élément central de l'identité régionale (Dantas 1988). Nous soutenons que c'est précisément la place accordée au Noir dans la construction de la culture et de l'identité de l'État et de la région Nord-Est qui autorise à considérer les offensives de l'EURD, dans les tribunaux de Bahia, comme des actes de discrimination et non comme un simple exercice de la liberté d'expression - comme c'est le cas dans d'autres États brésiliens ainsi qu'en Uruguay. La construction narrative dominante de l'histoire - ici régionale - constitue une ressource culturelle souvent reprise par les pratiquants de ces religions et qui semble également influencer, « de façon naturelle », les fonctionnaires qui entament des actions en justice contre l'EURD, ainsi que les juges qui les examinent et les journalistes qui les diffusent. Cela va dans le sens des thèses soutenues par Frigerio (2002a) qui aflirme que ces religions ont été principalement valorisées en tant que formes de "résistance culturelle » d'un groupe ethnique, revêtant une importance au niveau régional. Elles n'ont pas encore réussi cependant à se légitimer en tant que telles, comme un héritage spirituel ayant une valeur propre - comme par exemple l'hindouisme ou le bouddhisme, considérés comme des patrimoines spirituels de l'humanité. Dans les États brésiliens où ces religions ne sont pas considérées comme un bien culturel " ethnique », l'outil juridique a été dédaigné par les pratiquants qui envisagent l'entrée dans la politique locale comme un instrument plus efficace pour faire contrepoids au pouvoir croissant de l'EURD dans ce domaine, obtenant des soutiens au moins auprès du pouvoir législatif. L'exemple de Porto Alegre est sans doute à cet égard le plus représentatif.

En ce qui concerne les autres pays considérés ici, il apparaît clairement que l'intensité de la réponse aux attaques de l'EURD a été beaucoup plus forte en Uruguay qu'en Argentine. Dans les deux pays, la tournure prise par les conflits au Brésil ces dernières années - et particulièrement ce qui s'est passé à Porto Alegre en juin 2003 - a été déterminante pour le changement qualitatif qui s'est opéré au sujet des préoccupations exprimées. Ainsi, les informations concernant la possible participation politique de l'EURD dans les deux pays (sensiblement plus concrètes en Uruguay) ont joué le rôle de catalyseur supplémentaire pour l'intensification des ripostes.

Cependant, celles-ci n'ont pu voir le jour que grâce à l'existence préalable, chez au moins quelques leaders umbandistas des deux pays, d'un cadre interprétatif d'action collective qui met l'accent sur leurs droits civils. Cela est particulièrement évident lorsqu'on considère que le groupe de leaders uruguayens, dont les actions répondaient principalement à un cadre religieux/culturel, n'a pris aucune initiative contre cette Église, alors que c'est bien le groupe qui orientait effectivement son action en fonction de ce cadre de droits civils qui a adopté plusieurs 
stratégies de dénonciation des activités diffamatoires de l'EURD. En Argentine, la mère-de-saint qui s'est montrée le plus préoccupée par la question a aussi été celle qui a proposé, et défendu avec plus de force, le cadre des droits civils.

Le mouvement uruguayen se trouve non seulement dans une phase ascendante de son processus de légitimation, mais il peut également mobiliser des ressources culturelles dont ne disposent pas leurs collègues argentins. Cela se manifeste par les nombreuses et continuelles références à leur condition d'Uruguayens et, par là même, de citoyens, avec tous les droits qu'elle leur confère. Dans leurs lettres aux autorités de l'exécutif, du législatif et du judiciaire, comme dans leurs discours face aux médias, ils peuvent autant se réclamer de cette image classique de la nation uruguayenne, mettant en valeur sa tradition de respect et de sauvegarde des droits des minorités (Guigou 2003), que tirer parti d'une construction narrative nationale légèrement plus multiculturaliste, apparue ces dernières années, suite à la Conférence contre le racisme de Durban et aux efforts de l'organisation Mundo Afro qui reconnaît, notamment, l'existence et les apports culturels du segment noir de la population uruguayenne (Ferreira 2003). Cette construction narrative nationale, dans cette nouvelle version - encore naissante ou dans sa version plus traditionnelle, leur permet à la fois de revendiquer de manière plausible leur condition de citoyens uruguayens - ayant le droit constitutionnel d'exercer leur religion - qui repose sur une tradition nationale de respect envers les minorités et d'accuser les pasteurs de l'EURD d'être des «étrangers intolérants » qui violent les lois et les traditions locales de respect envers les autres citoyens.

En Argentine, au contraire, la construction narrative nationale dominante, caractérisée, comme le signalent plusieurs auteurs (Shumway 1991), par une mythologie d'exclusion de la différence et qui ne laisse aucune place aux expressions culturelles noires dans l'histoire argentine, limite les revendications des umbandistas argentins au lieu de les servir. Bien qu'ils aient essayé, à travers leurs congrès, leurs publications ou leurs interventions dans les médias, de présenter une " contre-narrative » de cette même histoire qui mette en valeur les apports des Noirs à la culture nationale, leurs efforts n'ont eu qu'une portée très limitée (Frigerio 2002a). Les accusations qui circulent depuis les années 1980, selon lesquelles les " pais » seraient principalement des sorciers, les excluent également d'une nation qui se considère non seulement homogène et européenne, mais aussi moderne et surtout " rationnelle », au sein de laquelle il n'y a donc aucune place - et par conséquent pas de droits civils - pour ceux qui pratiquent la magie (Frigerio 1996). Par conséquent, pour les umbandistas locaux, revendiquer leur statut de citoyens argentins ou faire appel à une tradition locale de respect envers les minorités s'avère être un argument culturellement inefficace.

Contrairement au Brésil, où son pouvoir politique lui a conféré un certain degré de légitimité sociale, en Uruguay et en Argentine, l'EURD est stigmatisée et n'est pas parvenue à établir des alliances locales. Les autres Églises évangélistes ne 
la reconnaissent pas comme une des leurs et la critiquent dès qu'elles en ont l'occasion, peut-être encore davantage en Argentine où ces Églises ont acquis, ces dernières années, un degré important d'union et de légitimation sociale ${ }^{20}$. Avec pour toile de fond les récents événements survenus au Brésil, on craignait qu'en associant le pouvoir politique au pouvoir économique qu'elle avait déjà, l'EURD ne sorte de son ostracisme local et n'attaque plus efficacement les pratiquants des religions afro-brésiliennes.

Pour certains spécialistes, l'EURD pourrait être vue comme une "afrobrésilianisation " de la religion pentecôtiste qui, en attaquant chaque fois plus l'umbanda, lui ressemble de plus en plus : inversement, ses offensives contre cette religion au Brésil, en Uruguay et en Argentine auraient transformé ses adversaires qui sont devenus, à leur tour, un peu plus semblables à elle. De fait, à partir du succès de l'EURD dans le champ politique, les umbandistas de quelques États brésiliens et des pays du Plata, d'habitude réticents à s'engager dans ce champ, envisagent la voie politique comme la meilleure manière de se défendre. Par ailleurs, les attaques de l'EURD dans les trois pays ont renforcé l'union et les contacts entre les leaders des religions afro-brésiliennes du Cône sud qui, principalement via internet, s'échangent des informations sur la situation du conflit et se soutiennent mutuellement dans les stratégies adoptées. Cela va dans le sens des thèses soutenues par Oro (1997), lorsqu'il affirirme que l'un des effets de la " guerre sainte » en question est le processus d'« umbandisation » de l'Église universelle et d'institutionnalisation des religions afro-brésiliennes. En d'autres termes, plus l'EURD et les religions afro se battent entre elles, plus elles se rapprochent l'une de l'autre. Ce qui signifie que l'EURD et les religions afro-brésiliennes sont constamment dans une relation dialectique d'antagonisme et de rapprochement, tels deux miroirs qui se reflètent mutuellement, le chemin emprunté par l'un étant éclairé par la lumière de l'autre. *

* Manuscrit reçu en mars 2005, accepté pour publication en juin 2005.

\section{NOTES}

1. " Mãe de santo " est le nom par lequel on désigne une femme à la tête d'un temple des religions afro-brésiliennes (lorsque le chef est un homme, il sera appelé " pai de santo »). " Encosto ", d'après les pratiquants de ces religions, est le nom donné à l'esprit d'un mort qui « s'attache » à un individu, vivant en lui et entrainant toute sorte de problèmes dans sa vie. En redéfinissant les « mães de santo » en tant que " mães de encosto », les pasteurs de l'Église universelle du royaume de Dieu (EURD) s'approprient des concepts issus des religions afro-brésiliennes, connus de tous, tout en les utilisant contre ces dernières religions, dans la mesure où ils suggèrent que leurs leaders n'invoquent pas des entités spirituelles bénéfiques mais maléfiques.

2. "L'offensive néo-pentecôtiste : le savon à la main, en condamnant l'umbandismo ".

3. Dans la plupart des temples des religions afro-brésiliennes du sud du Brésil et, surtout, de l'Uruguay et de l'Argentine, on pratique conjointement l'umbanda (y compris la quimbanda et le culte 
aux exus) et le batuque ou, sur une échelle moindre, le candomblé. En raison de cette pratique conjointe, les adeptes s'identifient de plus en plus comme « afro-umbandistas », bien que la majorité de la société - qui méconnaît les variations internes - les appelle de façon générale « umbandistas ». Lorsque nous utilisons ici l'expression " umbandistas ", nous le faisons dans son acception la plus large qui prend en compte les pratiquants de toutes ces variations religieuses. Pour l'Uruguay, nous utiliserons surtout le terme " afro-umbandistas » et, pour l'Argentine, " umbandistas/africanistas », étant donné qu'il s'agit là des expressions les plus employées par les pratiquants de ces deux pays.

4. Benford et Snow (2000, p. 614) définissent les " cadres d'action collective " (collective action frames) comme un « ensemble de croyances et de significations orientées vers l'action, qui inspirent et légitiment les activités et les campagnes d'organisation de mouvements sociaux ». Dans les études sur les mouvements sociaux, on considère que ces cadres postulent la « situation d'injustice que l'on désire combattre ", son " origine » et les " mesures » nécessaires pour la dépasser (Hunt, Benford et Snow 1994). Ils proposent également des "stratégies et des tactiques d'action collective " pour attirer l'attention sur le problème et essayer d'imposer leurs solutions (Snow et Benford 1992). Enfin, ils précisent qui sont les "destinataires de l'action " et identifient les « alliés " potentiels ainsi que les principaux "adversaires» (Hunt, Benford et Snow 1994). Carozzi (1998) et Frigerio (2003), entre autres, ont signalé que ces concepts sont aussi opérationnels dans l'étude des mouvements religieux. Williams (1995, p. 127) a suggéré la nécessité d'analyser les liens entre les cadres d'action collective et le répertoire culturel de la société concernée, auquel les mouvements sociaux peuvent emprunter leurs significations et symboles, utilisés dans leurs efforts rhétoriques. Selon cet auteur (ibid.), les « ressources culturelles " sont les outils symboliques utilisés par les mouvements dans leurs tentatives de changement social, qu'ils s'agissent d'idéologies formelles ou d'actions symboliques expressives.

5. Les nations sont des communautés imaginées, artefacts culturels devant être construits à travers des discours (Anderson 1983 ; Bhabha 1990). Selon Frigerio (2002a, p. 294) « les constructions narratives nationales dominantes fournissent une définition essentialiste d'identité nationale, insistant sur ses limites externes et sa composition interne, proposant l'agencement correct et ordonné des éléments (ethniques, religieux, de genre) qui la constituent, redéfinissant le présent au fur et à mesure qu'elles construisent un passé légitimant. Comme elles ne sont ni univoques ni indiscutables, elles peuvent être confrontées à des contre-discours ou sujettes à des lectures contestataires, en rencontrant plus ou moins de succès et d'acceptation sociale selon les différents contextes historiques ».

6. Nous utilisons le terme «transnationalisation » dans le sens donné par Badie et Smouts (1995, p. 70), à savoir « toute relation qui, par volonté délibérée ou par destination, se construit dans l'espace mondial au-delà du cadre étatique national et qui se met en cuvre en échappant, au moins partiellement, au contrôle ou à l'action médiatrice des États ».

7. L'Église universelle compte aujourd'hui près de deux millions de fidèles au Brésil et environ 600000 en dehors du Brésil. Elle a fondé, rien qu’au Brésil, 7000 temples et formé 14000 pasteurs. Dixsept grandes « cathédrales » sont en cours de construction dans des métropoles brésiliennes. L'Église est également propriétaire de plusieurs entreprises, dans les secteurs du tourisme, de la construction, de l'assurance, dans les finances et les médias. Dans ce dernier secteur, en particulier, elle possède deux chaînes de télévision, un journal au Brésil et sept à l'étranger, 62 chaînes de radio, maisons de graphisme, d'édition, de disques, de production audiovisuelle, et un portail sur internet (Mariano 2003 ; Fonseca 2003). De plus, l’Église universelle a une présence importante dans la politique brésilienne.

8. Le " descarrego ", que nous traduisons ici par " décharge », correspond au rituel d'élimination ou d'expulsion des « mauvaises énergies ».

9. Cette classification des religions afro-brésiliennes suit ce qui se passe au Rio Grande do Sul et dans les pays du Plata. Néanmoins, dans d'autres régions du Brésil, on trouve des possibilités de classification aussi bien du point de vue " émique » qu' " éthique ». Il existe une différence importante entre le reste du Brésil et l'extrême sud en ce qui concerne le culte et la représentation de la quimbanda, considérée comme une pratique rituelle à part entière et acceptée favorablement par les pratiquants dans le sud, tandis que, dans d'autres régions du Brésil, elle est surtout une catégorie d'accusation, associée aux pratiques de magie noire. 
10. Pour une description du batuque de Porto Alegre, voir Corrêa (1992) et Oro, éd. (1994). Brown (1986) et Negrào (1996) apportent une vision panoramique de l'umbanda. Sur le développement de l'umbanda en Uruguay, voir Hugarte (1998) et Cisneros (1995). Pour une analyse de la transnationalisation des religions afro-brésiliennes dans le Cône sud, voir Oro (1999) et Frigerio (2002a, 2002b, 2004).

11. Ce qui ne signifie pas qu'ils soient les seuls ou les plus populaires des pères ou mères-de-saint uruguayens. Selon la perspective adoptée dans ce texte, celle de l'analyse des mouvements sociaux, l'important est que ces leaders mènent des actions collectives qui modifient la relation entre les " umbandistas » et la société globale (pour plus de précisions sur cette perspective et son application détaillée au cas de l'umbanda en Argentine, voir Frigerio 2003).

12. En 1994, ces leaders réussirent à faire installer une statue de Yemanjá en face du Rio de la Plata, près de la plage où est réalisée la fête annuelle en l'honneur de cet orixá. Dans les années 1990, au fur et à mesure que le cadre culturel se substitue au cadre religieux, les réunions de chefs de culte pour arriver à l'union religieuse sont remplacées par des congrès sur la culture et la religion des peuples africains et afro-américains, auxquels participent des pères et des mères-de-saint, des anthropologues et des artistes afro-uruguayens. Les activités réalisées incluent des représentations de danses d'orixás, des présentations de livres sur l'umbanda et le batuque, des expositions de peinture sur les orixás, une projection de vidéos sur les religions afro-cubaines, un défilé d'habits religieux traditionnels et une exposition d'objets et d'autels religieux dans un musée d'anthropologie.

13. Le « Gran Buenos Aires » désigne les banlieues de la ville de Buenos Aires, situées au-delà du découpage administratif correspondant à la "capitale fédérale ».

14. Dans cette deuxième étape de mobilisation collective, le congrès « religieux », dominant dans la période précédente, est remplacé par le congrès "culturel » qui ne compte plus seulement sur les exposés de pères et mères-de-saint, mais aussi sur ceux de fonctionnaires, sociologues, intellectuels ou Afro-Argentins. Les cérémonies publiques d'umbanda, réalisées lors des précédents événements et incluant des transes de médiums aussi bien argentins et uruguayens, ont été transformées en des représentations scéniques de danses d'orixás, effectuées par des activistes culturels noirs d'origine brésilienne ou cubaine. Des démonstrations de capœira, rumba, danses de carnaval afro-bahianais ou de candomblé afro-uruguayen ont également été réalisées dans ces congrès. Les événements les plus sophistiqués ont organisé, en plus des danses, des expositions de peintures haïtiennes et/ou afrouruguayennes, de photos artistiques ou de reproductions matérielles d'autels et d'habits d'orixás. Ces représentations visuelles et performatives illustraient ce qu'était la culture africaine dont parlaient les intellectuels invités. 2003).

15. "Pais-de-santo e evangélicos da Bahia travam "guerra-santa" " (Jornal do Brasil, du 13 janvier

16. Données publiées dans une note intitulée "No para de subir : la Iglesia Universal del Reino de Dios (Pare de sufrir) acelera su expansión en Uruguay en medio de denuncias de todo tipo » (El Pais, supplément Qué Pasa, du 5 juin 2004).

17. En Uruguay, le Parti Colorado et le Parti Blanco se sont historiquement succédé à la tête du gouvernement national. Dans la dernière décennie, l'Encuentro Progresista-Frente Amplio (plus à gauche que les deux partis traditionnels) est devenu une troisième option politique. Son candidat a été élu président de la République en octobre 2004.

18. "L'Église universelle et son rejet des umbandistas : les attaques ne cessent pas en Uruguay ".

19. Le terme " despacho » fait référence aux offrandes de nourritures, de restes d'animaux sacrifiés, de bougies, adressées aux orixás ou aux exus par des membres des religions afro-brésiliennes. Les pratiques magiques, ayant pour but d'aider ou bien de porter préjudice à leurs destinataires, sont en revanche appelées " travaux ".

20. L'EURD a essayé à plusieurs reprises d'intégrer le conseil national évangéliste - une union de trois fédérations représentant une grande partie des Églises de cette obédience en Argentine -, mais elle n'a pas été admise (Hilario Wynarczyk communication personnelle). 


\section{RÉFÉRENCES BIBLIOGRAPHIQUES}

Almeida Ronaldo de

2003 "La guerre des possessions », in André Corten, Jean-Pierre Dozon et Ari Pedro Oro, éds, Les nouveaux conquérants de la foi. L'Église universelle du royaume de Dieu, Karthala, Paris, pp. 257-272.

ANDERSON Benedict

1983 Imagined communities : reflections on the origins and spread of nationalism, Verso, Londres-New York.

\section{BADIE Bertrand et Michel C. Smours}

1995 Le retournement du monde. Sociologie de la scène internationale, Presse de la Fondation nationale des sciences politiques/Dalloz, Paris.

BENFORD Robert et David SNow

2000 "Framing processes and social movements : an overview and assessment », Annual Review of Sociology, 26, pp. 611-639.

ВНАВНА Homi

1990 "Introduction : narrating the nation », in Homi Bhabha, éd., Nation and narration, Routledge, Londres, pp. 1-7.

Brown Diana

1986 Umbanda : religion and politics in urban Brazil, University of Michigan Press, Ann Arbor.

CARozzi María J.

1998 «El concepto de marco interpretativo en el estudio de movimientos religiosos ", Sociedad y Religión, 16-17, pp. 33-52.

\section{Cisneros Mariel}

1995 "¿Quién contó a los umbandistas?», Sociedad y Religión, 13, pp. 127-150.

\section{CORrÊA Norton}

1992 O Batuque do Rio Grande do Sul, EDUFRGS, Porto Alegre.

Corten André, Jean-Pierre Dozon et Ari Pedro Oro, éds

2003 Les nouveaux conquérants de la foi. L'Église universelle du royaume de Dieu, Karthala, Paris.

Cox Harvey

2003 "Préface ", in André Corten, Jean-Pierre Dozon et Ari Pedro Oro, éds, Les nouveaux conquérants de la foi. L'Église universelle du royaume de Dieu, Karthala, Paris, pp. 5-7.

\section{Dantas Beatriz Gois}

1988 Vovó Nagô e papai branco : usos e abusos da Africa no Brasil, Graal, Rio de Janeiro. 
FerReIra Luis

2003 El movimiento negro en Uruguay (1988-1998) y avances en Uruguay postDurban, Ediciones étnicas/Mundo Afro, Montevideo.

FONSECA Alexandre

2003 "Un empire médiatique », in André Corten, Jean-Pierre Dozon et Ari Pedro Oro, éds, Les nouveaux conquérants de la foi. L'Église universelle du royaume de Dieu, Karthala, Paris, pp. 213-230.

Freston Paul

2003 "L'Église universelle du royaume de Dieu en Asie. Le défi de l'universalité », in André Corten, Jean-Pierre Dozon et Ari Pedro Oro, éds, Les nouveaux conquérants de la foi. L'Église universelle du royaume de Dieu, Karthala, Paris, pp. 167-192.

Frigerio Alejandro

1996 "The medicalization of new religious movements in Argentina : cultural themes and deviance designations ", communication présentée à la $58^{\mathrm{e}}$ Réunion annuelle de l'Association for the Study of Religion, New York, 15-17 août.

2002a "Outside the nation, outside the diaspora : accommodating race and religion in Argentina ", Sociology of Religion, 63 (3), pp. 291-315.

2002b «La expansión de religiones afrobrasileñas en Argentina : representaciones conflictivas de cultura, raza y nación en un contexto de integración regio-

nal ", Archives des Sciences Sociales des Religions, 117, pp. 127-150.

$2003 \quad$ " " Por nuestros derechos ahora o nunca !" : construyendo una identidad colectiva umbandista en Argentina », Civitas. Revista de Ciências Sociais, 3 (1), pp. 35-68.

2004 "Re-africanization in secondary religious diasporas : constructing a world religion ", Civilizations, 51 (1-2), pp. 39-60.

Frigerio Alejandro et Ari Pedro Oro

1998 "Sectas satánicas" en el Mercosur», Horizontes Antropológicos, 8, pp. 114-150.

Frigerio Alejandro et Hilario WyNARCZYK

2004 «Cult controversies and government control of new religious movements in Argentina (1985-2001) », in James Richardson, éd., Regulating religion : case studies from around the Globe, Kluwer Publishing, New York, pp. 453475 .

GiumbelLi Emerson

2002 O fim da religião. Dilemas da liberdade religiosa no Brasil e na França, Attar Editorial/CNPq/Pronex, Sào Paulo.

Guigou Nicolás

1993 «Luchas religiosas y confrontación: una aproximación al conflicto pentecostalismo-umbanda ", communication présentée aux $I I T^{c}$ Jornadas sobre alternativas religiosas en Latinoamérica, Buenos Aires.

2003 La nación laica : religión civil y mito-praxis en el Uruguay, Ediciones La Gotera, Montevideo. 


\section{Hugarte Renzo Pi}

1992 "La iglesia pentecostal Dios es Amor en el Uruguay », Cuadernos de Antropologia, 9, pp. 63-94.

1998 Los cultos de posesión en Uruguay : antropología e historia, Facultad de Humanidades/Ed. Banda Oriental, Montevideo.

\section{Hunt Scott, Robert BENFord et David SNow}

1994 "Identity fields : framing processes and the social construction of movement identities ", in Enrique Laraña, Hank Johnston et Joseph Gusfield, éds, New social movements : from ideology to identity, Temple University Press, Philadelphia, pp. 185-208.

MACEDO Edir

1987 Orixás, Caboclos e Guias : deuses ou demônios ?, Universal Produções, Rio de Janeiro.

1995 O diablo e seus anjos, Ed. Gráfica Universal, Rio de Janeiro.

MARIANO Ricardo

1995 Neopentecostalismo : os pentecostais estão mudando, mémoire de maîtrise, FFLCH/USP, Sao Paulo.

2003 "Le royaume de prospérité de l’Église universelle ", in André Corten, Jean-Pierre Dozon et Ari Pedro Oro, éds, Les nouveaux conquérants de la foi. L'Église universelle du royaume de Dieu, Karthala, Paris, pp. 197212.

NEGRÃo Lísias

1996 Entre a cruz e a encruzilhada, EDUSP, São Paulo.

OLIVEN Ruben

1992 A parte e o todo, Vozes, Petrópolis.

\section{Oro Ari Pedro}

1989 «Immigrants européens et religions afro-brésiliennes dans le sud du Brésil ", Archives des Sciences Sociales des Religions, 68 (1), pp. 125-140.

1997 "Neopentecostais e afro-brasileiros : quem vencerá esta guerra ? ", Debates do NER, 1, pp. 10-36.

1999 Axe Mercosul, Vozes, Petrópolis.

2004 «La transnationalisation du pentecôtisme brésilien : le cas de l'Église universelle du royaume de Dieu ", Civilisations, 51 (1-2), pp. 155-170.

Oro Ari Pedro, éd.

1994 As religiōes a fro-brasileiras do Rio Grande do Sul, EDUFRGS, Porto Alegre.

PRANDI Reginaldo

1991 Os candomblés de São Paulo, Hucitec, São Paulo.

2003 "As religiōes afro-brasileiras e seus seguidores ", Civitas. Revista de Ciências Sociais, 3 (1), pp. 15-34.

\section{Shumway George}

1991 The invention of Argentina, University of California Press, Berkeley. 
JOURNAL DE LA SOCIÉTÉ DES AMÉRICANISTES

\section{SNow David et Robert BENFORD}

1992 "Master frames and cycles of protest ", in A. Morris et C. McClurg Mueller, éds, Frontiers in social movement theory, Yale University Press, New Haven, pp. 133-155.

Williams Rhys $\mathrm{H}$.

1995 "Constructing the public good : social movements and cultural resources ", Social Problems, 42 (1), pp. 124-144. 